\title{
Complex Obstetric Perineal Injury Reconstruction Using Antropyloric Valve Transposition
}

\author{
Saket Kumar, Noushif Medappil, Sunil Kumar Singh, Abhijit Chandra \\ Department of Surgical Gastroenterology, King George’s Medical University, Lucknow, India
}

Despite significant advancements in the field of medicine, management of complex obstetric perineal injuries remains a challenge. Although several surgical techniques have been described, no techniques have provided satisfactory long-term results. Recently, a perineal transposed antropyloric valve has been used for anorectal reconstruction in patients with damaged or excised anal sphincters. We describe this technique in the case of complex obstetric perineal trauma with extensive tissue loss, presenting with end stage fecal incontinence. The functional outcome after this procedure was evaluated. The patient tolerated the surgery well, and there were no procedure-related upper gastrointestinal disturbances. Short-term functional outcomes were encouraging. At the 36-month follow-up, the patient's neoanal resting and squeeze pressures were 50 and $70 \mathrm{mmHg}$, respectively. The postoperative St. Mark's incontinence score was 7. Perineal antropyloric valve transposition is feasible and can be successfully applied in the management of end-stage fecal incontinence associated with complex obstetric perineal injury.

Keywords: Anal sphincter; Fecal incontinence; Obstetric injury; Rectovaginal fistula

\section{INTRODUCTION}

Obstetric perineal laceration with ano-rectal sphincter (ARS) disruption remains the most common cause of fecal incontinence (FI) among women worldwide. Management of complex perineal trauma has been a subject of extensive research since no current treatment options have provided effective long-term results [1]. The overlapping sphincteroplasty, which is considered the standard surgical procedure for Obstetric Anal Sphincter Injuries (OASIS), has disappointing results in the long-term $[1,2]$. Recently, a perineal transposed antropyloric valve (APV) for total anorectal reconstruction has been described for excised or severely damaged ARS as an alternative to permanent colostomy [3]. To our knowledge, the present report is the first to describe

Received: April 22, 2018 - Accepted: August 21, 2018

Correspondence to: Abhijit Chandra, MCh

Department of Surgical Gastroenterology, King George's Medical University, Lucknow, UP, India

Tel: +9199335920302, Fax: +91-522-2256116

E-mail: abhijitchandra@hotmail.com

ORCID: https://orcid.org/0000-0002-3940-7428

(C) 2020 The Korean Society of Coloproctology

This is an open-access article distributed under the terms of the Creative Commons Attribution NonCommercial License (https://creativecommons.org/licenses/by-nc/4.0) which permits unrestricted noncommercial use, distribution, and reproduction in any medium, provided the original work is properly cited. the use of transposed APV for perineal reconstruction in a case of complex OASIS.

\section{CASE REPORT}

A 24-year-old primipara presented to us with a 3-week-old thirddegree perineal tear and OASIS. The delivery was conducted at a remote primary health care center; an episiotomy was not performed. The patient had developed complete FI following injury. Because of the injury severity, a primary repair of the perineal laceration was not attempted. A diverting loop colostomy was created one week after delivery, and the patient was referred to us for definitive management. The patient was otherwise healthy and had no comorbidities or any significant past medical or surgical history. The patient was psychologically distressed by the loss of her child and because of the newly created abdominal stoma. General and systemic examinations were unremarkable. She weighed approximately $60 \mathrm{~kg}$ and had a body mass index of 24 $\mathrm{kg} / \mathrm{m}^{2}$. On perineal examination, anal tone was diminished and a large communication between the rectum and vagina could be visualized $2 \mathrm{~cm}$ above the anal verge. A per-vaginal speculum examination revealed a fistulous opening on the posterior wall with surrounding mucosal erythema. Communication could be easily demonstrated by a per-rectal examination as well (Fig. 1). Three- 


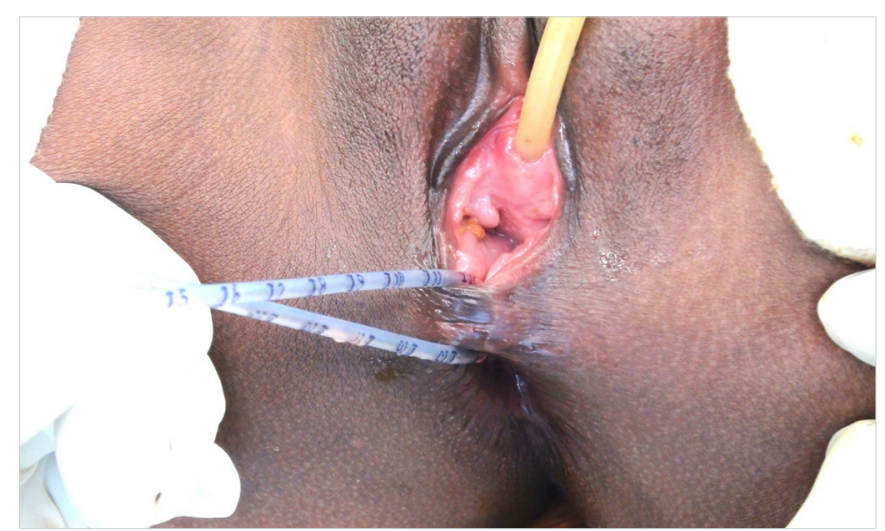

Fig. 1. A preoperative image of the perineum in the lithotomy position showing a fistulous communication between the lower rectum and vagina along with a thinned rectovaginal septum.

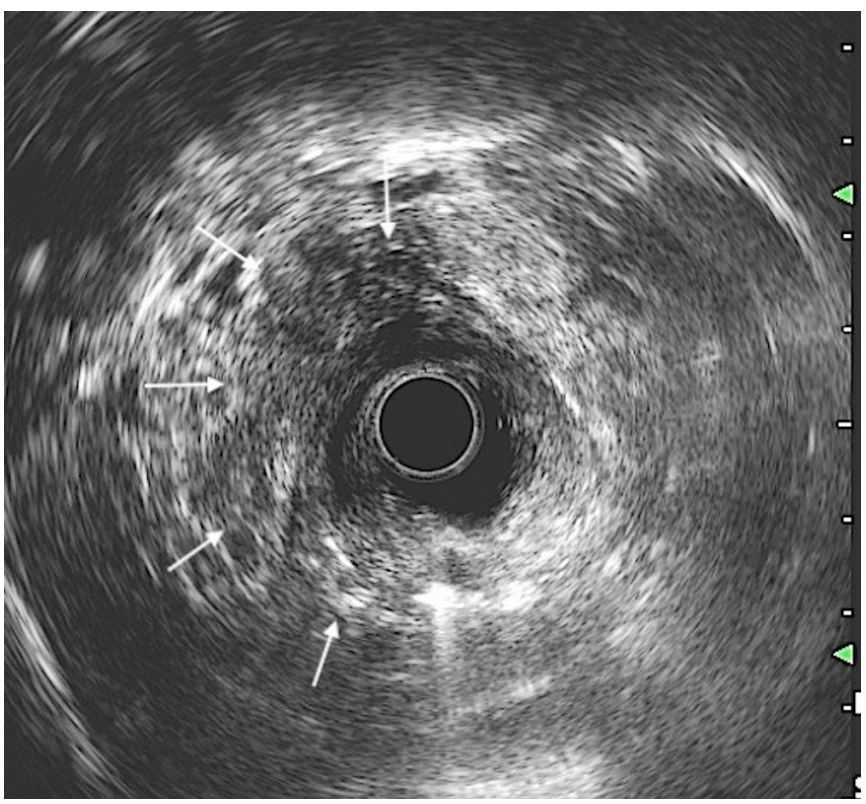

Fig. 2. Three-dimensional endoanal ultrasound examination performed in the lithotomy position showing extensively damaged (arrows) anal sphincter in the anterolateral position.

dimensional endoanal ultrasound was conducted, which showed severely damaged ARS (both internal and external sphincters, $>180^{\circ}$ ) in the anterolateral position (Fig. 2).

Because of the irreparably damaged ARS, the patient was offered APV transposition for anal reconstruction. We conducted this procedure in compliance with the principles of the Declaration of Helsinki. The operative protocol was reviewed and approved by the Institutional Review Board (IRB) of the King George's Medical University, Lucknow, Uttar Pradesh, India (IRB No. 1206/ Rcell-10 dated 18-08-2010). Written informed consent was obtained from the patient. The surgery was performed under spinal

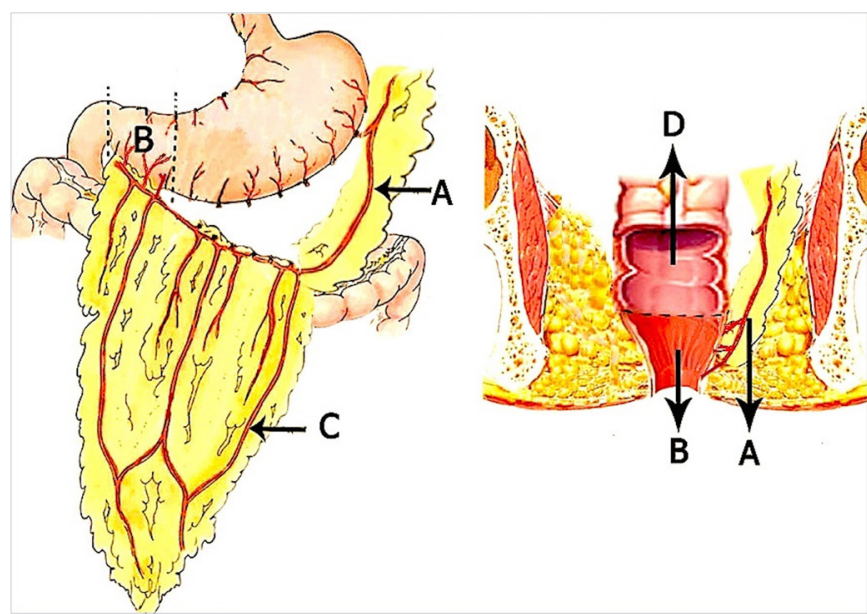

Fig. 3. Schematic diagram of the abdominal and perineal procedures (left panel) abdominal and (right panel) perineal. A, left gastroepiploic arterial pedicle; $\mathrm{B}$, antropyloric segment; $\mathrm{C}$, gastrocolic omentum; D, descending colon.

anaesthesia in the lithotomy position. A midline laparotomy incision was created. Gastrocolic omentum was mobilised close to the transverse colon. Hepatic flexure of the colon was mobilized and the duodenum was kocherized. Branches of the gastroepiploic arcade to the greater curvature of the stomach were serially ligated and divided, which safeguarded the terminal branches to the antropyloric region. The right gastroepiploic artery was divided, which preserved the infrapyloric branches. The APV was then mobilized based on the left gastroepiploic pedicle after dividing its antral and duodenal ends. Pelvic dissection was initiated by removing the intrapelvic adhesions, and the rectum was mobilized to the pelvic floor. The fistulous communication between the rectum and vagina was identified and deconstructed. The defect on the vaginal side was closed with polyglactin 2-0 sutures in a single continuous layer. The rectum was divided at the fistula level, and the edges were debrided. The antral end of the mobilized APV segment was then sutured to the proximal rectal end with interrupted silk 2-0 sutures (Fig. 3). The graft was invaginated through the distal rectal cuff, and the duodenal end was sutured to the freshened perianal skin. A movie file has been provided to illustrate the steps of the procedure. The proximal colostomy was retained. Bowel continuity was maintained with retrocolic posterior gastrojejunostomy. Postoperative recovery was fairly unremarkable, and the patient had clinically good neoanal sphincter tone. The patient had mild surgical wound infection, which was managed conservatively. Oral feeding was initiated gradually, and the patient was discharged 9 days after surgery. A clinical and neoanal manometric examination performed at the 1-month follow-up displayed good sphincter pressures with a resting pressure of 58 $\mathrm{mmHg}$ and maximum squeeze pressure of $74 \mathrm{mmHg}$ (Fig. 4). A distal colonic loop barium retention study was performed through the colostomy that showed a smooth beak impression of 


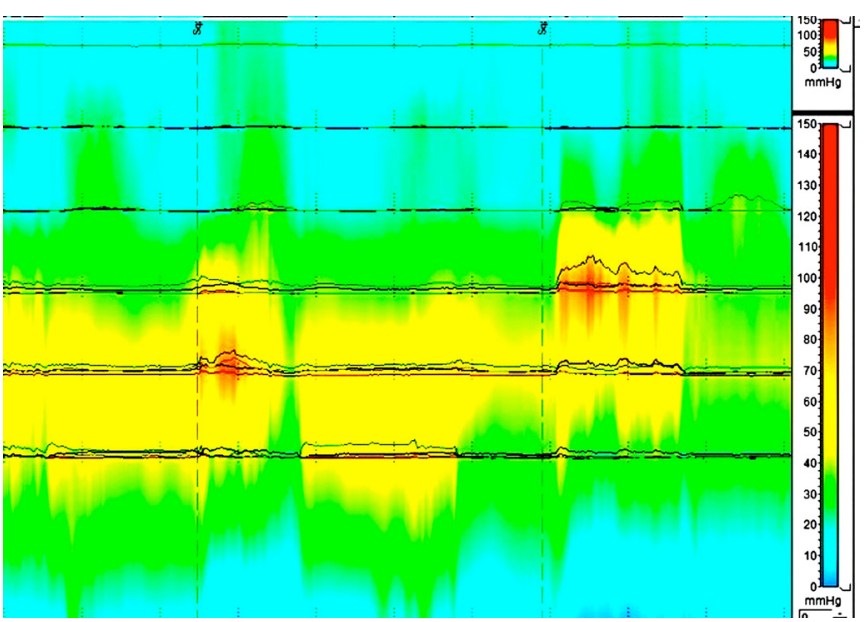

Fig. 4. Manometry tracing showing the constant resting tone of the transposed antropyloric segment (yellow).

the APV graft with holding of barium proximal to it on late films (Fig. 5). The diverting colostomy was closed 3 months after surgery. One month after colostomy closure, the patient's St. Mark's incontinence score was seven, which indicates good sphincter control. The patient was followed-up for 36 months during which the patient remained satisfied with the surgery outcome and maintained a fairly good continence status. The patient developed no long-term complications related to gastrectomy. Persistent mucous discharge from the duodenal end of the graft was initially a problem but gradually subsided over time.

\section{DISCUSSION}

Globally, OASIS is the leading cause of FI among young women. It has been estimated that between $4 \%$ and $6 \%$ of women having a vaginal delivery suffer from FI [4]. Commonly, these injuries occur due to a prolonged second stage of labor, which results in pressure necrosis of the rectovaginal septum. The patient in this study had obstructed labor that resulted in severe perineal laceration with a rectovaginal fistula and resulted in the death of the patient's baby.

The incidence of underlying ARS injury in patients with obstetric perineal trauma and rectovaginal fistula is estimated to be very high [2]. Therefore, meticulous sphincter evaluation in these patients using endo-rectal ultrasound and/or manometric study is justified [5]. The patients clinically present with fecal, gaseous, or mucus discharge through the vagina along with symptoms of FI.

Management of complex obstetric perineal injury still remains a challenge and often requires specialized and multidisciplinary care. Currently, surgical treatment with overlapping sphincteroplasty is considered the standard of care. Though the reported short-term results of this procedure are good (74\% of improved continence at three months), the long-term outcome is subopti-
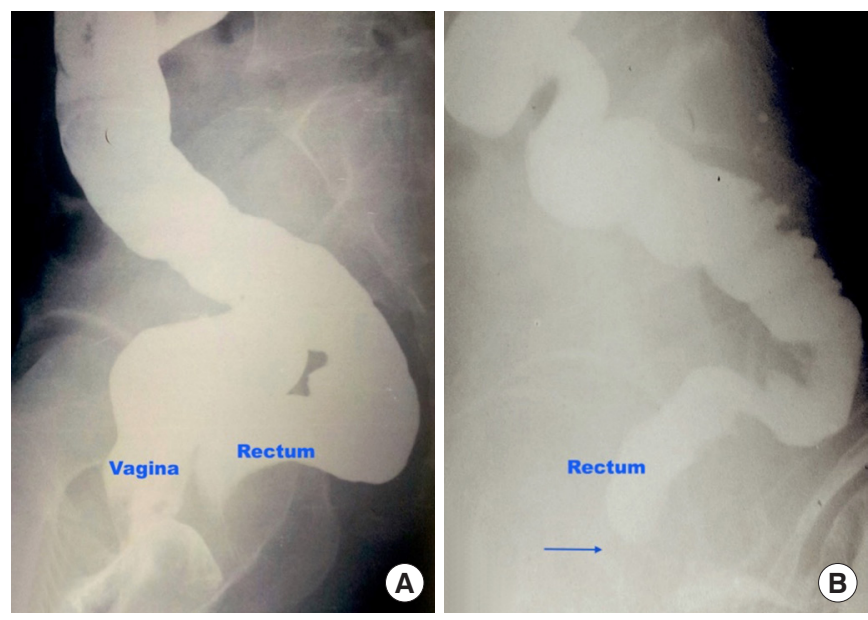

Fig. 5. (A) Preoperative distal colon loop barium study showing the rectovaginal fistulous connection. (B) Postoperative film showing retention of contrast (arrow) proximal to the perineal antropyloric valve.

mal. Larger studies with follow-ups of more than 5 years have consistently shown that only half of the patients undergoing sphincteroplasty remained continent in the long-term $[6,7]$. Furthermore, in our patient sphincteroplasty was not suitable as the sphincter was irreparably damaged and only native sphincter tissue fibrosis was present. In similar situations when the anal sphincter is severely damaged, skeletal muscles (gracilis, gluteus maximus) have also been used as wraps around the anus to augment ARS function, but with less satisfactory outcomes $[8,9]$. Dynamic graciloplasty (converting type II to type I fibers) and artificial bowel sphincter implantation have also been attempted, but with limited success and significant associated morbidity $[9$, 10]. The role of sacral nerve stimulation in the management of traumatic FI is being explored and has been found to be effective in patients with partial ARS tears [11]. Still today, there are no satisfactory options for anal reconstruction that are an alternative to a permanent colostomy in patients with severely damaged ARS.

Permanent colostomy may offer good long-term quality of life (QoL) for patients with end-stage FI, although data are lacking from the developing world. Living with a permanent colostomy is an undesired outcome due to the social stigma in Asia. Moreover, the inability to receive proper stoma care along with the life-long need to purchase stoma appliances are issues that make colostomy unfavorable [12]. In the present case, when the patient was given a choice, she readily opted for perineal reconstruction with APV transposition.

Perineal transposition of APV is a novel technique for total anorectal reconstruction in patients with severely damaged/excised ARS complex. It is hypothesized that this fatigue resilient smooth muscle segment may provide an appropriate substitute for native ARS because prolonged tonic contraction is provided [13]. In a recently published study on initial outcomes of APV transposition in patients with severe FI, most of the patients showed an in- 
crease in anal manometric pressures with improvements in their postoperative incontinence and QoL scores [3]. The transposed antropylorus behaves like an obstructive ring at the perineal outlet but lacks voluntary control. In our previous study, the postoperative functional result and QoL were better in patients with an intact rectum and sensate anal canal compared to the excision group [3]. Moreover, the gluteus maximus is a strong and bulky muscle that acts as a natural accessory anal contractor. Many patients gradually learn to use their gluteus muscle as an accessory mechanism for voluntary fecal control.

The potential long-term complications of gastrojejunostomy (dumping syndrome, downstream acid injury, and alkaline reflux syndrome) remain a valid concern, though the actual incidence has been reported as low $[3,14]$. Our patient did not have any of these complications in the postoperative follow-up. Initially the patient had to apply perineal pads due to persistent mucus discharge from the duodenal end of the graft, which gradually decreased over time. At the 3-year follow-up, the patient had fairly good continence status (St. Mark's incontinence score of 7) and overall procedure-related satisfaction.

Perineal APV transposition can be an effective alternative to a permanent colostomy in patients with complex obstetric perineal injury and associated ARS damage. This technique, though feasible and effective in the preliminary evaluation, is technically demanding and needs to be performed in a surveillance setting. Lack of long-term results and the complex nature of surgery may preclude routine use of this procedure at present, although it is a promising technique that opens a new dimension in the management of intractable FI.

\section{CONFLICT OF INTEREST}

No potential conflict of interest relevant to this article was reported.

\section{ACKNOWLEDGMENTS}

We thank Mr. Vikas Srivastava for preparing the schematic diagram for the article.

\section{REFERENCES}

1. Altomare DF, Binda GA, Dodi G, La Torre F, Romano G, Rinaldi
$\mathrm{M}$, et al. Disappointing long-term results of the artificial anal sphincter for faecal incontinence. Br J Surg 2004;91:1352-3.

2. Debeche-Adams TH, Bohl JL. Rectovaginal fistulas. Clin Colon Rectal Surg 2010;23:99-103.

3. Chandra A, Kumar A, Noushif M, Gupta V, Singh D, Kumar M, et al. Perineal antropylorus transposition for end-stage fecal incontinence in humans: initial outcomes. Dis Colon Rectum 2013; 56:360-6.

4. Keighley MR, Bick D, MacArthur C. Prevalence and obstetric factors in childbirth-related fecal incontinence (meeting abstract). Dis Colon Rectum 1995,38:14.

5. Tsang CB, Madoff RD, Wong WD, Rothenberger DA, Finne CO, Singer D, et al. Anal sphincter integrity and function influences outcome in rectovaginal fistula repair. Dis Colon Rectum 1998; 41:1141-6.

6. Barisic GI, Krivokapic ZV, Markovic VA, Popovic MA. Outcome of overlapping anal sphincter repair after 3 months and after a mean of 80 months. Int J Colorectal Dis 2006;21:52-6.

7. Halverson AL, Hull TL. Long-term outcome of overlapping anal sphincter repair. Dis Colon Rectum 2002;45:345-8.

8. Sonnino RE, Reinberg O, Bensoussan AL, Laberge JM, Blanchard H. Gracilis muscle transposition for anal incontinence in children: long-term follow-up. J Pediatr Surg 1991;26:1219-23.

9. Devesa JM, Vicente E, Enríquez JM, Nuño J, Bucheli P, de Blas G, et al. Total fecal incontinence--a new method of gluteus maximus transposition: preliminary results and report of previous experience with similar procedures. Dis Colon Rectum 1992;35:339-49.

10. Baeten CG, Konsten J, Spaans F, Visser R, Habets AM, Bourgeois IM, et al. Dynamic graciloplasty for treatment of faecal incontinence. Lancet 1991;338:1163-5.

11. Boyle DJ, Knowles CH, Lunniss PJ, Scott SM, Williams NS, Gill KA. Efficacy of sacral nerve stimulation for fecal incontinence in patients with anal sphincter defects. Dis Colon Rectum 2009;52: 1234-9.

12. Bansal V, Bhutani R, Doval D, Kumar K, Pande P, Kumar G. Neo adjuvant chemo-radiotherapy and rectal cancer: can India follow the West? J Cancer Res Ther 2012;8:209-14.

13. Chandra A, Ghoshal UC, Gupta V, Jauhari R, Srivastava RN, Misra A, et al. Physiological and functional evaluation of the transposed human pylorus as a distal sphincter. J Neurogastroenterol Motil 2012;18:269-77.

14. Tack J. Gastric motor disorders. Best Pract Res Clin Gastroenterol 2007;21:633-44. 